\title{
Assessing shelf life of injectable biologicals in ready-to-administer containers
}

\author{
Mike Allwood, Alan-Shaun Wilkinson
}

\section{EXTENDING THE SHELF LIFE OF INJECTABLE BIOLOGICALS: WHAT EVIDENCE IS REQUIRED?}

We are all familiar with the issues concerning the assessment of the stability of chemical drug entities. We now face a new challenge as the number of injectables with active biological chemical entities grows rapidly. The availability of data to support the extension of shelf lives of monoclonal antibodies (mAbs), for example, is sparse and universally incomplete. The difficulty lies in confirming the retention of bioactivity and the detection of species that could be immunogenic to the patient, such as aggregates or subvisible particulates after reconstitution and dilution in ready-toadminister delivery containers. The formation of increased amounts of aggregates on storage or handling can be a direct indication of the stability of the product. Often small amounts of dimer are present within the original biological product at the point of manufacture. However, the amount of aggregate can change at the expense of active monomer, depending on storage, dilution conditions and stability of the product. This must be monitored using appropriate techniques, such as size exclusion chromatography and light scattering.

While there are limited chemical data in the literature which suggest that proteinbased drugs may be relatively resistant to detectable physical and gross chemical changes, there is as yet no valid bioassay evidence to support the extension of the shelf lives of such products. The most

University of Derby, Derby, UK

Correspondence to Professor Mike Allwood, 16 Rectory Lane, Breadsall, Derby DE21 5LL, UK; allwoodmichael1@sky.com recent guidelines, based on a European Consensus Conference to consider guidelines for the practical stability studies of anticancer drugs, ${ }^{1}$ recognise the importance of biological evidence to support the shelf life of biological products. It also warns that stability testing of biological products is not as straightforward as that for their chemical counterparts; the latter have well characterised chemical structures that are simple to analyse. The official guidance documents (ICH Q2 R1 (analytical validation); ICH Q5C (stability testing of biotechnological/ biological products) and ICH Q6B (specifications test procedures and acceptance criteria for biotechnological/biological products)) that govern stability testing procedures are typically aimed at licensing of new drug products and do not provide a road map for how to analyse or interpret biological product data. There appears to be no single analytical assay for determining the stability of these products and a suite of tests is required, including tests for chem$\mathrm{ical} /$ physical stability and biological potency or activity. Due to the more complex chemical structures associated with biological products it is not recommended to use accelerated/stressed stability testing, for example, forced degradation through heating would be inappropriate as a test for stability of a mAb-based product. This is because most protein-based products will denature and unfold from their active form as they are heated close to the melt temperature (referred to as the Tg or glass transition temperature).

This process is essentially irreversible. Similarly, extreme excursions in $\mathrm{pH}$ (high and low) will adversely affect the product which relies on charged groups (and polar groups) at the surface maintaining their charge and overall isoelectric point ( $\mathrm{pI}$ ) for solubility. The requirement for a suitable biological assay with biological products is obvious, but this needs to be specific for that biological product and ideally should be a cellular-based assay rather than a simple binding assay such as that afforded in an ELISA. In specific cases when the product has an enzymatic function relating to clinical efficacy, a biochemical assay may be appropriate. However, generally biochemical assays are not reliable reporters of biological potency.

Finally, when multiple mechanisms of action contribute to clinical efficacy it may prove necessary to apply more than one biological cellular assay. Each cellular assay should be validated and shown to correlate with in vivo biological activity if practicable. As with any assay design, appropriate controls should be incorporated to validate that the assay is working correctly and should include the use of positive reference standards in addition to negative controls. One resource text on the development and application of cellular assays is by Uma Prabhakar and Marian Keller. ${ }^{2}$

In summary, the challenge presented by biologicals is a formidable one when it comes to stability testing. However, it is one that can and is being addressed by the application of specialist knowledge combining a range of analytical and cellularbased approaches.

Competing interests None.

Provenance and peer review Commissioned; internally peer reviewed.

To cite Allwood M, Wilkinson A-S. Eur J Hosp Pharm 2013;20:146.

Published Online First 26 January 2013

Eur J Hosp Pharm 2013;20:146.

doi:10.1136/ejhpharm-2012-000251

\section{REFERENCES}

1 Bardin C, Astier A, Vulto A, et al. Guidelines for the practical stability studies of anticancer drugs: a European consensus conference. Eur J Hosp Pharm 2012;19:278-85.

2 Prabhakar U, Keller M. Validation of Cell-based Assays in the GLP Setting: A Practical Guide. Chichester: Wiley-Blackwell, 2008. 